\title{
Technè
}

La science au service de l'histoire de l'art et de la préservation des biens culturels

46 | 2018

Science et conservation

\section{Conservation des mousses polyuréthane ester : consolidation par application de mélanges de polysiloxanes}

Conservation of polyurethane foams: consolidation through the application of polysiloxane mixtures

Lucille Royan, Céline Daher, Nathalie Balcar, Gilles Barabant et Agnès Lattuati-Derieux

\section{OpenEdition}

\section{Journals}

Édition électronique

URL : http://journals.openedition.org/techne/536

DOI : $10.4000 /$ techne.536

ISSN : 2534-5168

Éditeur

C2RMF

Édition imprimée

Date de publication : 1 décembre 2018

Pagination : 119-125

ISBN : 978-2-11-152829-1

ISSN : 1254-7867

\section{Référence électronique}

Lucille Royan, Céline Daher, Nathalie Balcar, Gilles Barabant et Agnès Lattuati-Derieux, «Conservation des mousses polyuréthane ester : consolidation par application de mélanges de polysiloxanes », Technè [En ligne], 46 | 2018, mis en ligne le 19 décembre 2019, consulté le 24 juillet 2020. URL : http:// journals.openedition.org/techne/536 ; DOI : https://doi.org/10.4000/techne.536

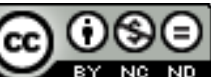

La revue Technè. La science au service de l'histoire de l'art et de la préservation des biens culturels est mise à disposition selon les termes de la Licence Creative Commons Attribution - Pas d'Utilisation Commerciale - Pas de Modification 4.0 International. 
Lucille Royan

Céline Daher

Nathalie Balcar

Gilles Barabant

Agnès Lattuati-Derieux

\section{Conservation des mousses polyuréthane ester : consolidation par application de mélanges de polysiloxanes}

Conservation of polyurethane foams: consolidation through the application of polysiloxane mixtures

Résumé. Parmi les objets présents dans les collections d'art $d u X X^{e}$ siècle, ceux réalisés en mousse de polyuréthane (PUR) ester posent de sévères problèmes de conservation liés principalement à la perte de leurs propriétés mécaniques due à leur dégradation par hydrolyse. Le but de ce travail a été de développer et tester un mélange de polymères de la famille des polysiloxanes qui permettrait de stabiliser les mousses PUR ester : un mélange à base d'AMDES (3-aminopropylmethyldiethoxysilane), hydrophile, et d'OTMS (octyltriméthoxysilane), hydrophobe. L'application de ce mélange suivant différents modes se voulant transposables à de véritables objets a été testée sur des échantillons de mousse PUR ester par des restaurateurs du patrimoine.

Mots-clés. Consolidation, mousses polyuréthanes ester, polysiloxanes, méthodes d'application, conservation-restauration.

\begin{abstract}
Among the objects present in collections of 20th-century art, those made of ester polyurethane foam pose serious conservation problems, mainly related to the loss of their mechanical properties due to their breakdown through hydrolysis. The aim of this work was to develop and test a mix of polymers from the polysiloxanes family which would enable ester polyurethane foams to be stabilised: a mixture based on hydrophilic AMDES (3-aminopropylmethyldiethoxysilane) and hydrophobic OTMS (octyltrimethoxysilane). The application of this mixture in different manners intended to be transposable to real objects was tested on polyurethane foam samples by national heritage restors.

Keywords. Consolidation, polyurethane foams, polysiloxanes, application methods, conservation-restoration.
\end{abstract}

\section{Introduction}

Depuis les années 1960, les mousses polyuréthanes, initialement utilisées à des fins industrielles, ont rapidement eu la faveur des artistes et designers qui les ont employées dans la réalisation de sculptures ${ }^{1}$, mobiliers ${ }^{2}$, textiles et accessoires de design et de mode.

Il existe deux types de mousses polyuréthanes (PUR) : le type éther et le type ester. Elles peuvent présenter des dégradations assez prononcées, d'une vitesse d'apparition rapide - une vingtaine d'années en moyenne - en fonction de la qualité de la fabrication et des conditions de conservation. La dégradation du PUR éther est principalement liée à son oxydation, accélérée par la lumière et la température, dont résultent un brunissement et une friabilité de surface. Les mousses polyuréthanes ester sont, quant à elles, sensibles à l'humidité, et leur dégradation, qui se déclenche au cœur du matériau, se traduit par une perte de résilience, des fissurations, un effritement ainsi qu'un jaunissement important.
Ainsi, la prise de conscience d'un véritable problème en matière de conservation de ces mousses polyuréthanes utilisées dans les objets patrimoniaux est en constante évolution depuis les années 1990. Si plusieurs types de traitements de consolidation sur des œuvres en mousse de PUR éther avaient été testés et appliqués ${ }^{3}$ avec ou sans ${ }^{4}$ succès, toutes les solutions de consolidation de matériaux poreux en polyuréthane ester proposées présentaient des inconvénients majeurs, comme des changements importants de couleurs, de texture et/ou de tenue mécanique, jusqu'à un travail de recherche soutenu en 2012 par Pellizzi et $a l^{5}$.

Ce travail introduit l'utilisation de consolidants de type polysiloxanes - plus précisément des aminoalkylalkoxysilanes (AAAS) - pour la consolidation de mousses PUR ester industrielles modernes, qui ont déjà prouvé leur grande efficacité pour le renforcement du papier ${ }^{6}$. Le 3-aminopropylmethyldiethoxysilane (AMDES) a permis d'apporter, grâce à la formation d'un polymère associé au réseau du polyuréthane, un renfort mécanique sans modification des propriétés

Lucille Royan, restauratrice, MNAM-Centre Georges Pompidou (lucille.royan@centrepompidou.fr). Céline Daher, post-doctorante, Centre de recherche sur la conservation (CRC, USR 3224), muséum national d'Histoire naturelle, ministère de la Culture et de la Communication, CNRS (celine.daher@mnhn.fr). Nathalie Balcar, ingénieure d'étude, C2RMF (nathalie.balcar@culture.gouv.fr). Gilles Barabant, chef de la filière xx siècle-art contemporain, département Restauration, C2RMF (gilles.barabant@culture.gouv.fr). Agnès Lattuati-Derieux, ingénieure de recherche, C2RMF (agnes.lattuati-derieux@culture.gouv.fr). 

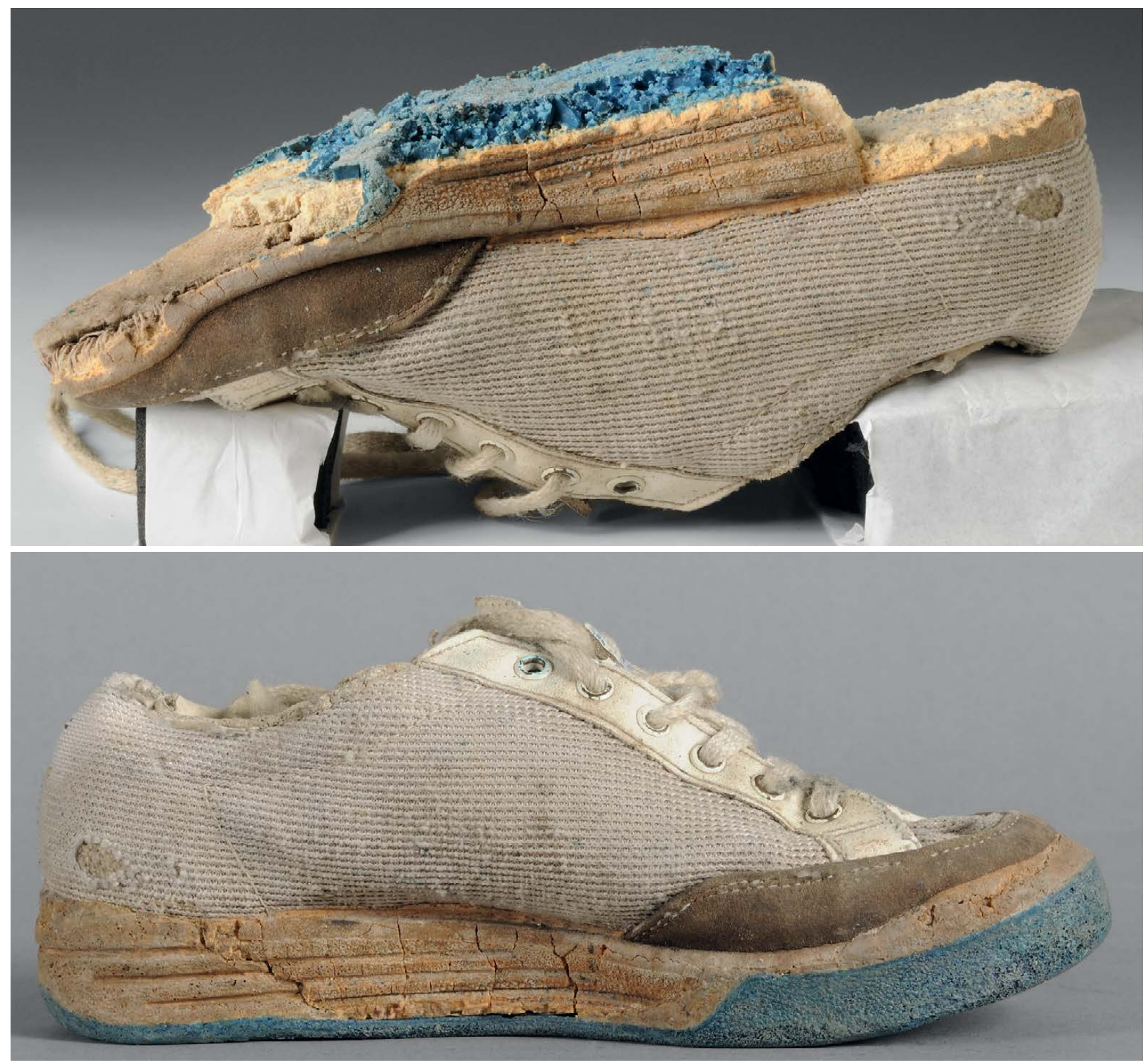

Fig. 1. Vue avant (en haut) et après (en bas) : traitement de consolidation et de réintégration de la semelle de la chaussure appartenant à l'œuvre In the spirit of Fluxus de Ben Vautier, conservée au musée d'Art moderne de la Ville de Paris. ( Inp/G. Vanneste. 
visuelles des mousses ${ }^{7}$. Cette association chimique entre la mousse et le consolidant en fait un traitement non réversible, devant être utilisé à des fins essentiellement curatives, pour prolonger la conservation d'un objet très altéré. Cependant, le caractère hydrophile de la mousse traitée, dû à la présence d'un groupement amine, ne permet pas au traitement de jouer son rôle de barrière de protection pour ces mousses sensibles à l'humidité ; et peut même entraîner un risque d'hydrolyse supplémentaire. Par ailleurs, le mode d'application du consolidant alors défini, l'immersion, n'étant pas adapté pour le traitement de la très grande majorité des objets d'art, un travail de recherche mené dans le cadre d'un diplôme de restaurateur du patrimoine ${ }^{8}$ avait permis d'évaluer la possibilité d'une application plus contrôlée et localisée à la semelle en PUR ester dégradée d'une chaussure appartenant à l'œuvre In the spirit of Fluxus de Ben Vautier, conservée au musée d'Art moderne de la Ville de Paris (fig. 1).

Si l'état de dégradation très avancé de l'objet étudié avait conduit au choix de sa consolidation pour ne pas perdre davantage de matière, la pénétration du consolidant, de quelques millimètres seulement, avec la technique de pulvérisation choisie, n'était pas totalement satisfaisante et son caractère hydrophile ne pouvait présager d'une stabilité optimale dans le temps.

Inscrit dans la suite de ces avancées, notre travail a été réalisé dans le cadre du projet PURESCOP, financé en 2014 par la Fondation des Sciences du Patrimoine/LabEx PATRIMA, mené au Centre de recherche sur la conservation (CRC) et en collaboration avec le C2RMF et le LPPI (Laboratoire de Physico-Chimie des Polymères et Interfaces, EA 2528). Le but de celui-ci était de développer une technique de consolidation curative plus performante que celle précédemment proposée, en déterminant, au sein de la même famille des polysiloxanes, un consolidant ou un mélange de consolidants qui serait plus adapté à la sensibilité du matériau PUR ester, et en définissant un ou des modes d'application permettant une pénétration homogène et en profondeur du consolidant dans la mousse.

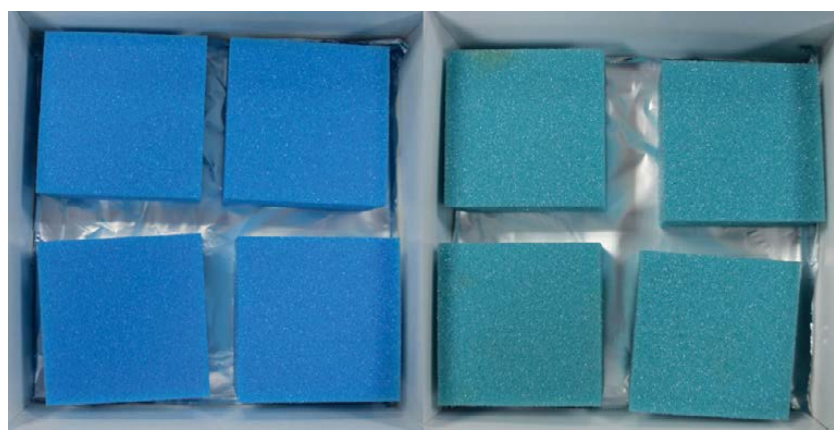

Fig. 2. Échantillons de mousses PUR ester avant et après vieillissement en enceinte climatique. Un jaunissement (de bleu à vert) des mousses est observé. () C. Daher.

\section{Sélection des consolidants}

\section{Choix des échantillons supports}

Des échantillons de mousse PUR ester industrielle moderne bleue (mousse souple à cellules ouvertes de densité de $30 \mathrm{~kg} /$ $\mathrm{m}^{3}$ ) ont été artificiellement vieillis (fig. 2) en enceinte climatique pendant 3 semaines à $90^{\circ} \mathrm{C}$ et $50 \%$ d'humidité relative et sans lumière, conditions considérées comme conduisant à des échantillons de mousses représentatifs de mousses naturellement dégradées par hydrolyse. Les mousses ont ensuite été conservées à l'abri de la lumière à $23{ }^{\circ} \mathrm{C}$ et $50 \%$ d'humidité.

\section{Choix des consolidants}

Pour cette étude, un consolidant hydrophobe trifonctionnel a été sélectionné. Il s'agit de l'octyl-triméthoxysilane (OTMS) auquel les chaînes alkyles à 8 atomes de carbone confèrent un caractère hydrophobe pouvant limiter la présence d'eau sur les mousses PUR ester, et donc limiter leur altération hydrolytique. Le caractère trifonctionnel permet de former un réseau polymère tridimensionnel, gage de bonne tenue mécanique du consolidant. Cependant, l'absence de groupement hydrophile rend ce composé très peu réactif à l'état pur $^{10}$, c'est-à-dire que l'humidité présente dans l'air ne suffit pas à amorcer sa polymérisation. Il a donc été nécessaire de le mélanger à un composé qui possède un groupement réactif, l'AMDES. Différents mélanges avec des proportions croissantes d'OTMS dans l'AMDES (OTMS/AMDES, en \%vol) ont été étudiés : 10/90, 25/75, 50/50,75/25, 90/10, ainsi que les deux composés purs, $0 / 100$ et 100/0. Ces mélanges ont été préparés dans un solvant de même nature chimique, l'hexaméthyldisiloxane (HMDS). La concentration en consolidant a été limitée à 2,5\% et $10 \%$ dans l'HMDS afin que la solution présente une bonne viscosité et de manière à éviter une prise de masse trop importante des échantillons de mousse.

\section{Test d'efficacité des mélanges}

Dans un premier temps et afin de déterminer le (ou les) mélange(s) les plus efficaces, l'ensemble des échantillons a été traité par immersion, une technique facile à mettre en œuvre et reproductible ${ }^{11}$.

Différentes techniques d'analyses ont été mises en œuvre pour évaluer l'efficacité des mélanges. Les échantillons ont été pesés avant et après traitement pour déterminer leur prise de masse. Celle-ci doit être modérée afin de ne pas dénaturer les caractéristiques du matériau et, par transposition, de l'objet. Dans notre cas, elle se situe entre 15 et $20 \%$ pour une concentration de $2,5 \%$; et entre 50 et $80 \%$ pour une concentration de $10 \%$.

Des mesures d'angles de contact ${ }^{12}$ ont été effectuées sur l'ensemble des mousses traitées afin d'évaluer leur caractère 
hydrophile/hydrophobe. La mousse de référence non vieillie montre un angle de contact de $115^{\circ}$ environ, qui diminue légèrement quand la mousse est vieillie $\left(105^{\circ}\right)$. Les mousses traitées à l'AMDES seul $(0 / 100)$ et celles avec le mélange 10/90 absorbent la goutte d'eau sans permettre les mesures. Ces mélanges rendent les mousses trop hydrophiles et ne conviennent donc pas pour un traitement supposé hydrophobe. Le mélange 25/75 augmente l'angle de contact sur les mousses traitées à $130^{\circ}$ environ, et les mélanges avec de fortes proportions en OTMS (50/50, 75/25, $90 / 10)$ entraînent une hyper-hydrophobie des mousses $\left(145^{\circ}\right)$. Ces trois mélanges ont donc été retenus pour la suite des analyses.

Afin d'étudier la tenue mécanique des mousses traitées avec les différents mélanges, des essais par compression ont été effectués ${ }^{13}$. Les résultats obtenus (fig. 3) sur les mousses vieillies traitées montrent qu'une concentration de 2,5\% n'est pas suffisante pour une consolidation mécanique des mousses PUR, les courbes contrainte/déformation étant toutes proches de celles de la mousse de référence vieillie et non traitée. Une concentration de $10 \%$ améliore les propriétés mécaniques, le profil des courbes observé se rapprochant davantage de celui de la mousse de référence non vieillie et non traitée.

Enfin, des mesures colorimétriques ont été effectuées sur les échantillons de mousses à l'aide d'un spectrophotomètre (X-Rite) afin de vérifier que la présence du consolidant ne modifie pas ostensiblement les propriétés visuelles des mousses. Les mousses traitées avec des mélanges peu concentrés ne montrent pas de variations de couleurs significatives. La couleur des mousses vieillies traitées par des mélanges plus concentrés $(10 \%)$ évolue vers une couleur proche de celle de l'échantillon de mousse non vieillie (un décalage de 10 unités sur l'axe $\left.b^{*}\right)$. Cette variation résulte d'une diminution apparente du jaunissement des échantillons.

\section{Optimisation du mode d'application}

L'évaluation de différents modes d'application du mélange consolidant a ensuite été nécessaire afin d'optimiser la profondeur de pénétration, ainsi que l'homogénéité du dépôt. Ces modes d'application, testés sur les échantillons de mousses présentés précédemment, devaient pouvoir être utilisés pour des traitements de consolidation sur des surfaces altérées d'objets de collections patrimoniales de dimensions et de formes variées, possiblement composites et pouvant présenter des dégradations importantes.

L'appréciation des résultats reposant en premier lieu sur une observation visuelle, le consolidant a été coloré en rouge (colorant rouge Soudan). Pour mieux quantifier les distances de pénétration, une analyse en microscopie électronique à balayage (MEB) couplée à une analyse par spectroscopie à rayons X à dispersion d'énergie (EDS) a été effectuée. Les premiers essais d'application ont consisté en la projection de particules de tailles variables à la surface des mousses (avec un spray, un aérographe ou encore un nébuliseur). D’autres modes ont ensuite été mis en pratique, comme l'application au pinceau ou par dépôt de gouttes. L'ensemble de ces traitements ainsi que les perspectives envisagées à l'issue de ce travail sont présentés et discutés ici.

Nous avons appliqué le mélange 75/25, préparé deux heures en amont de la réalisation des tests d'application et mis en solution à $10 \%$ dans le solvant.

\section{Les modes d'application mis en ouvre}

\section{Vaporisation}

Dans un premier temps, des applications par projection de gouttelettes de diverses tailles via diverses méthodes spray, aérographe et nébulisation - ont été testées. Ces modes d'application précis et sans contact sont parfaitement adaptés aux différentes contraintes induites par la diversité des objets du patrimoine. Cependant, les résultats obtenus ne sont pas satisfaisants. En effet, le consolidant ne se dépose principalement qu'en surface, sur 200 à $300 \mu \mathrm{m}$ d'épaisseur, soit sur la première rangée de cellules de la mousse, ainsi qu'observé grâce aux images et cartographies EDS du silicium obtenues par MEB de coupes transversales d'échantillons (fig. 4).

\section{Application de gouttes de consolidant}

Des gouttes de $1 \mathrm{~mL}$ ont été déposées à la micropipette de façon régulière sur la totalité de la surface de l'échantillon (fig. 5 a). Au moment de l'application, la pénétration du consolidant ne semble pas homogène, le consolidant empruntant des chemins préférentiels dans la mousse. 

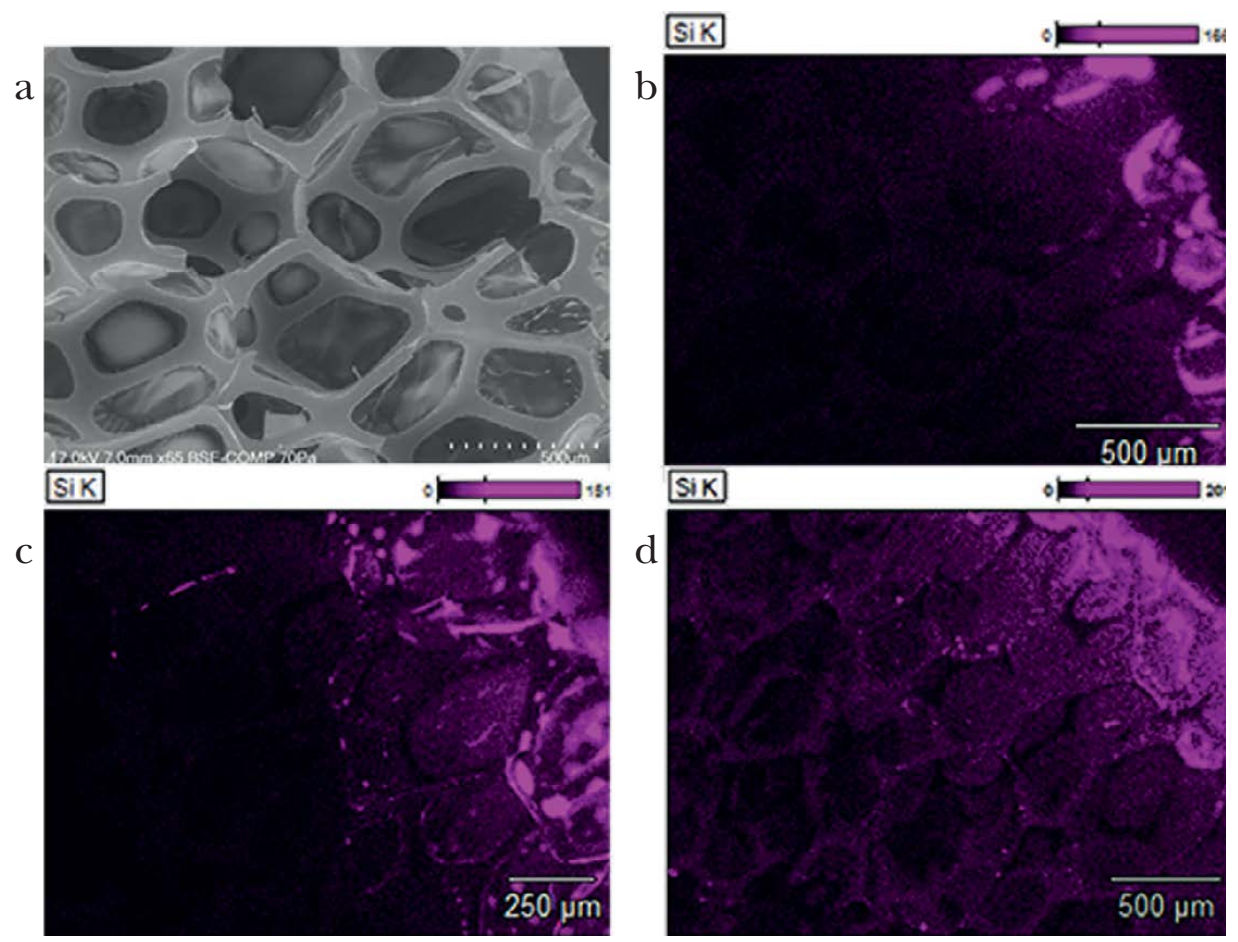

Cette méthode permet une pénétration de $8 \mathrm{~mm}$ environ en surface, mais aussi sur le côté opposé à la surface. En effet, une partie du consolidant coule rapidement à travers la mousse, puis stagne sur le fond de l'échantillon et remonte alors légèrement par capillarité. Après séchage, l'homogénéité du consolidant dans la mousse paraît plus satisfaisante, bien qu'une zone non consolidée reste visible au cœur de la mousse.

\section{Imprégnation par la solution de consolidant}

Le consolidant est versé sur un bâtonnet en verre qui canalise le liquide sur la mousse (fig. 5 b). L'échantillon est posé sur un tamis afin de recueillir le surplus de solution. La mousse est ensuite délicatement tournée sur tous ses côtés afin de bien répartir le mélange dans la mousse. Ceci est observé au moment de l'application et après séchage. Cependant, les angles et arêtes de la mousse dégradée sont altérés par la manipulation.

\section{Application au pinceau avec une interface fibreuse}

Afin de déposer le consolidant de la manière la plus homogène possible sur la mousse sans avoir à la manipuler, et afin d'éviter tout frottement du pinceau pouvant abraser la surface, une application au pinceau via une interface fibreuse (papier Japon) a été mise en place (fig. 5 c).

Comme avec la technique par application de gouttes, le consolidant pénètre la mousse de $8 \mathrm{~mm}$ environ sur chacun des côtés. L'interface ajoutée permet une application plus homogène du consolidant sur la surface de la mousse, bien qu'il emprunte, en pénétrant, des chemins préférentiels. Après séchage, la diffusion du consolidant dans la mousse ne semble pas plus homogène que pour les échantillons traités par application de gouttes. Le consolidant paraît avoir stagné sur les bords.

Les objets du patrimoine ne présentant que très rarement des surfaces horizontales, notamment dans le cas d'objets tridimensionnels en mousse, nous avons voulu observer comment la solution diffusait dans l'épaisseur du matériau lorsqu'elle est appliquée sur une surface verticale. Au moment de l'application au pinceau du consolidant, ce dernier semble majoritairement migrer vers le bas de la mousse. L'observation visuelle des coupes des échantillons montre que la pénétration dans l'épaisseur de la mousse n'est que de $1 \mathrm{~mm}$.

\section{Limites des modes d'application testés}

Pour toutes les techniques testées, le consolidant pénètre mais ne se répartit pas dans la mousse de façon homogène. En effet, la mousse étant constituée de cellules ouvertes de tailles variables, le liquide suit des chemins préférentiels. Le dépôt du consolidant à travers une interface fibreuse offre la possibilité d'une application homogène en surface, même s'il ne permet pas d'orienter la pénétration du produit. Cependant, étant donné qu'un objet composé entièrement ou en partie de mousse polyuréthane ester ne dispose que rarement d'uniques surfaces horizontales, comment est-il alors possible de consolider toute la mousse ?

L'option la plus satisfaisante pour faire pénétrer et diffuser le consolidant uniformément dans un échantillon de mousse consiste à le faire rouler sur ses trois faces non 
imprégnées après application du consolidant. Toutefois, cette technique n'est pas compatible avec des surfaces fragilisées. De plus, il semble difficile de transposer cette technique à un objet qui ne pourrait pas être manipulé en raison de ses dimensions, de son poids, de son état de conservation, etc.

\section{Conclusion}

Différents mélanges OTMS/AMDES peuvent être utilisés pour la consolidation des mousses PUR ester. Afin que le mélange de consolidants soit hydrophobe - condition considérée comme indispensable étant donné la très fréquente dégradation hydrolytique des PUR ester -, il est nécessaire de choisir un mélange composé d'au moins 50 \% d'OTMS. De plus, afin de retrouver des propriétés mécaniques et visuelles convenables sur une mousse dégradée, la concentration ne doit pas dépasser $10 \%$ dans le solvant.

À l'issue de ce travail, il semble difficile de déterminer une technique d'application du consolidant qui lui permette une pénétration homogène quels que soient les formes et les formats des objets et ne dégradant pas la surface d'une mousse altérée. La seule technique offrant un résultat satisfaisant nécessite une manipulation peu envisageable sur des objets du patrimoine.

Les échantillons traités par dépôt régulier de gouttes semblent, après séchage, être les plus uniformément consolidés malgré la présence, à cœur, de petites zones non ou peu consolidées. La consolidation de surfaces par les méthodes de vaporisation peut aussi être envisagée : la « coque » ainsi formée constitue-t-elle un obstacle pour l'humidité ambiante, protégeant l'ensemble de l'objet d'une hydrolyse ? Il serait intéressant d'évaluer le comportement des échantillons traités après un vieillissement artificiel, et de conclure sur la nécessité de consolider l'intégralité d'un volume de mousse polyuréthane ester dégradée.
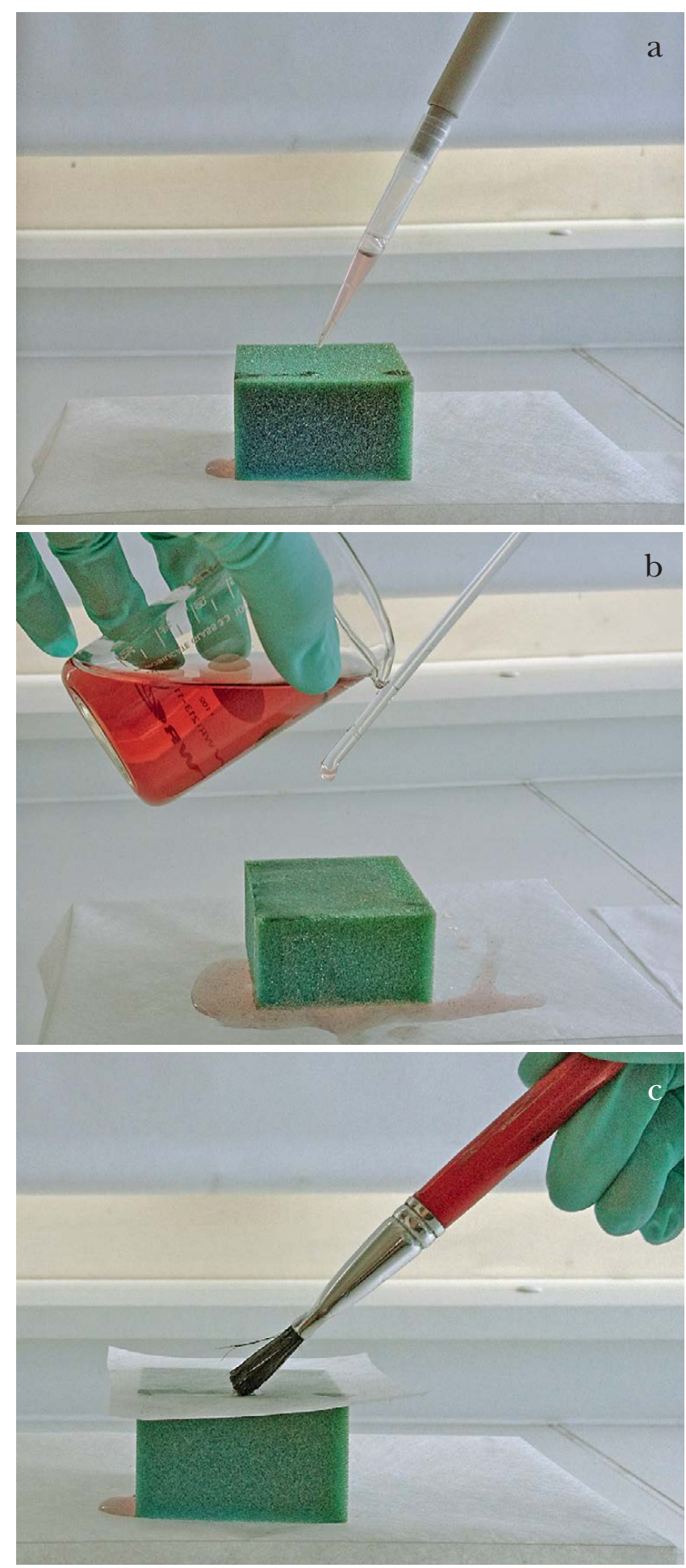

Fig. 5. Vues des techniques d'application par dépôt de gouttes (a), imprégnation (b), et au pinceau avec interface fibreuse (c). (C) C. Daher. 


\section{Remerciements}

Ce travail a été réalisé dans le cadre du projet PURESCOP financé en 2014 par la Fondation des Sciences du Patrimoine/ LabEx PATRIMA ANR-10-LABX-0094-01. Les auteurs souhaitent remercier O. Fichet, I. Fabre-Franck et S. Cantin du Laboratoire de Physicochimie des Polymères et des Interfaces, EA 2528, de l'université de Cergy-Pontoise pour leur collaboration sur les choix des consolidants et l'accès à l'appareil de mesure des angles de contact, ainsi que H. Cheradame (université d'Évry) pour ses conseils, S. Bouvet (Bibliothèque nationale de France) pour l'accès à la machine d'essais en compression, O. Belhadj (Centre de Recherche sur la Conservation, USR 3224) pour les mesures MEB et F. Duboisset pour sa collaboration à la réalisation des essais de consolidation.
Notes

1. 2. Pour exemples, Untitled de John Chamberlain, 1963-1965, la série des Expansions de César ; le siège Pratone, de la marque Gufram.

3. Van Oosten, 2011 ; Fröhlich, 2011.

4. Cas de «Larry the Lamb» marionnette de la série télévisée "Toytow » entièrement imprégnée de Dunlop ${ }^{\circledR}$, laque à base de caoutchouc, dont la couleur a évolué du jaune au brun-rouge, Waentig, 2008.

5. Pelizzi, 2012.

6. Dupont, 2010, Souguir, 2011.

7. Pelizzi, 2012.

8. Royan, 2013.

9. Pellizzi, 2014. Le choix s'est porté sur ces mousses dès le travail de thèse

d'Eleonora Pellizzi, car il s'agissait du seul fabricant pouvant fournir des mousses PUR ester prédécoupées à la demande.

10. Brochier-Salon, 2008.

11. Pellizzi, 2012a.

12. Mesures effectuées avec de l'eau ultra-pure et en dynamique (plan incliné, mesure de l'angle d'avancée).

13. Pellizzi, 2012b.

\section{Bibliographie}

Brochier Salon M.-C., Bayle P-A., Abdelmouleh M., Boufi S., Belgacem M.-N., 2008, "Kinetics of hydrolysis and self condensation reactions of silanes by NMR spectroscopy", Colloids and Surfaces A, 312 : 83-91.
Fröhlich R., 2011, "Pedicures for Pesce's 'IL PIEDE'”, dans FUTURE TALKS 009, The conservation of modern materials in applied arts and design, actes de colloque, Pinakothek der Moderne, Munich, 22-23 octobre 2009, The International Design Museum, Munich, p. 124-131.

Pellizzi E., Lattuati-Derieux A., d'Espinose de Lacaillerie J.-B., Lavédrine B., Cheradame H., 2012b, "Reinforcement properties of 3-aminopropylmethyldiethoxysilane and $\mathrm{N}$-(2-Aminoethyl)-3aminopropylmethyldimethoxysilane on polyurethane ester foam", Polymer Degradation and Stability, 97(11): 23402346.

Pellizzi E., Lattuati-Derieux A., Lavédrine B., Cheradame H., 2014, "Degradation of polyurethane ester foam artifacts: chemical properties, mechanical properties and comparison between accelerated and natural degradation", Polymer Degradation and Stability, 107: 255-161.

Royan L., Pellizzi E., 2015, “Application of aminoalkylalkoxysilane consolidant on polyurethane ester foam from a Ben Vautier's work of art", dans FUTURE TALKS 013, Lectures and Workshops on Technology and Conservation of Modern Materials in Design, actes de colloque, Pinakothek der Moderne, Munich, 22-24 octobre 2013, The International Design Museum, Munich, p. 67-73.
Souguir Z., Dupont A.-L., d'Espinose de Lacaillerie J.-B., Lavédrine B., Cheradame H., 2011, "Chemical and physicochemical investigation of an aminoalkylalkoxysilane as strengthening agent for cellulosic materials", Biomacromolecules, $12(6)$ : 2082-2091.

Van Oosten T., 2011, PUR facts, Conservation of Polyurethane foam in Art and Design, Amsterdam University Press, Amsterdam.

Waentig F., 2008, Plastics in Art, A study from the conservation point of view, Michael Imhof Verlag, Petersberg.

\section{Documents inédits}

Pellizzi E., 2012a, Étude du vieillissement des mousses de polyuréthane ester et consolidation par les aminoalkylalcoxysilanes, thèse de doctorat, université d'Évry-Val d'Essonne.

Royan L., 2013, "In the spirit of Fluxus" (19652003). Conservation-restauration d'une œuvre composite de Ben Vautier, musée d'Art moderne de la Ville de Paris. Étude du vieillissement d'une double semelle de chaussure en polyuréthane ester et recherche sur sa consolidation par les aminoalkylalkoxysilanes, mémoire de fin d'études, Institut national du patrimoine (spécialité sculpture), Paris. 